\title{
Two distinct halo populations in the solar neighborhood
}

\section{Lithium abundances ${ }^{\star}, \star \star$}

\author{
P. E. Nissen ${ }^{1}$ and W. J. Schuster ${ }^{2}$
}

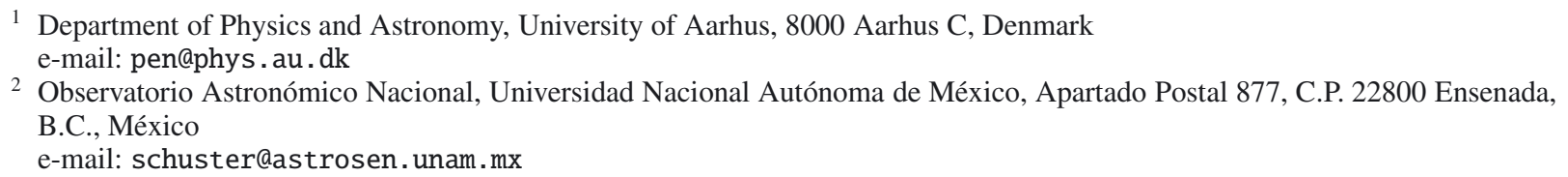

Received 4 April 2012 / Accepted 13 May 2012

\begin{abstract}
Context. A previous study of $\mathrm{F}$ and $\mathrm{G}$ main-sequence stars in the solar neighborhood has revealed the existence of two distinct halo populations with a clear separation in $[\alpha / \mathrm{Fe}]$ for the metallicity range $-1.4<[\mathrm{Fe} / \mathrm{H}]<-0.7$. Taking into account the kinematics and ages of the stars, some Galactic formation models suggest that the "high-alpha" halo stars were formed in situ, whereas the "lowalpha" stars have been accreted from satellite galaxies.

Aims. In this paper we investigate if there is a systematic difference in the lithium abundances of stars belonging to the high- and low-alpha halo populations.

Methods. Equivalent widths of the Li I 6707.8 A resonance line are measured from high resolution VLT/UVES and NOT/FIES spectra and used to derive Li abundances on the basis of MARCS model atmospheres. Furthermore, masses of the stars are determined from the $\log T_{\text {eff }}-\log g$ diagram by interpolating between evolutionary tracks based on Yonsei-Yale models.

Results. There is no significant systematic difference in the lithium abundances of high- and low-alpha stars. For the large majority of stars with masses $0.7<M / M_{\odot}<0.9$ and heavy-element mass fractions $0.001 \lesssim Z<0.006$, the lithium abundance is well fitted by a relation $A(\mathrm{Li})=a_{0}+a_{1} M+a_{2} Z+a_{3} M Z$, where $a_{0}, a_{1}, a_{2}$, and $a_{3}$ are constants. Extrapolating this relation to $Z=0$ leads to a lithium abundance close to the primordial value predicted from standard Big Bang nucleosynthesis calculations and the WMAP baryon density. The relation, however, does not apply to stars with metallicities below $[\mathrm{Fe} / \mathrm{H}] \simeq-1.5$.

Conclusions. We suggest that metal-rich halo stars were formed with a lithium abundance close to the primordial value, and that lithium in their atmospheres has been depleted in time with an approximately linear dependence on stellar mass and $Z$. The lack of a systematic difference in the Li abundances of high- and low-alpha stars indicates that an environmental effect is not important for the destruction of lithium.
\end{abstract}

Key words. stars: abundances - stars: atmospheres - stars: interiors - Galaxy: halo - early Universe

\section{Introduction}

The discovery by Spite \& Spite (1982a, 1982b) of a uniform lithium abundance in halo dwarf and subgiant stars with effective temperatures $T_{\text {eff }} \gtrsim 5700 \mathrm{~K}$ has led to important advances in the fields of stellar physics and Big Bang nucleosynthesis. Recent works on lithium abundances in very metal-poor stars raise, however, severe problems in our understanding of how lithium was synthesized in the Big Bang and subsequently destroyed in stars.

The work of Spite \& Spite (1982b) showed that eight halo stars with $5700<T_{\text {eff }}<6300 \mathrm{~K}$ and $-2.4<[\mathrm{Fe} / \mathrm{H}]<-1.4$ have an average lithium abundance $A(\mathrm{Li})=\log \left(N_{\mathrm{Li}} / N_{\mathrm{H}}\right)+12=2.06$ with a rms dispersion of 0.10 dex and no significant trends as a function of $T_{\text {eff }}$ or $[\mathrm{Fe} / \mathrm{H}]$. Later, this so-called "Spite plateau" has been confirmed for larger samples of halo stars (e.g. Molaro et al. 1995; Spite et al. 1996). More recently, a very precise

\footnotetext{
* Based on observations made with the Nordic Optical Telescope on La Palma, and on data from the European Southern Observatory ESO/ST-ECF Science Archive Facility (programs 65.L-0507, 67.D-0086, 67.D-0439, 68.D-0094, 68.B-0475, 69.D-0679, 70.D-0474, 71.B-0529, 72.B-0585, 76.B-0133 and 77.B-0507).

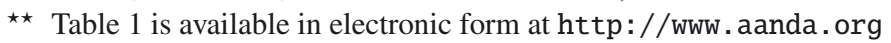

determination of lithium isotopic abundances by Asplund et al. (2006) showed $A(\mathrm{Li})$ to be constant at 2.23 in the metallicity range $-2.5<[\mathrm{Fe} / \mathrm{H}]<-1.4$ with a dispersion of 0.03 dex only. A revised $T_{\text {eff }}$-scale explains the higher Li-plateau abundance as compared to Spite \& Spite (1982b). Furthermore, Asplund et al. find that the bulk of lithium (i.e. more than $~ 95 \%$ ) consists of the ${ }^{7} \mathrm{Li}$ isotope.

Although the data of Asplund et al. (2006) support the existence of a Li-plateau from -2.5 to -1.4 in $[\mathrm{Fe} / \mathrm{H}]$, there is a hint of a decline of $A(\mathrm{Li})$ for five stars with $[\mathrm{Fe} / \mathrm{H}]<-2.5$. This is in agreement with an earlier investigation by Ryan et al. (1999), who found a dependence of $A(\mathrm{Li})$ on metallicity for a sample of 23 very metal-poor halo stars; $A(\mathrm{Li})$ declines from 2.15 at $[\mathrm{Fe} / \mathrm{H}]=-2.5$ to 2.03 at $[\mathrm{Fe} / \mathrm{H}]=-3.5$. Several recent works (Boesgaard et al. 2005; Bonifacio et al. 2007; Aoki et. al. 2009; Sbordone et al. 2010) also suggest a "melt-down" of the Spite plateau by finding a decrease and/or increased scatter in $A(\mathrm{Li})$ as a function of decreasing metallicity below $[\mathrm{Fe} / \mathrm{H}] \simeq-2.5$. In this connection, the work of Meléndez et al. (2010) is of particular interest. For a sample of 88 dwarf halo stars with $-3.5<[\mathrm{Fe} / \mathrm{H}]<-1.0$ having precise measurements of the equivalent width of the $6707.8 \AA \mathrm{Li}$ I line, they find a break in $A(\mathrm{Li})$ at $[\mathrm{Fe} / \mathrm{H}] \simeq-2.5$. Above this metallicity, the stars 
distribute around a plateau at $A(\mathrm{Li})=2.27$, whereas stars in the metallicity range $-3.5<[\mathrm{Fe} / \mathrm{H}]<-2.5$ have an average lithium abundance of $A(\mathrm{Li})=2.18$. This two-step distribution of $A(\mathrm{Li})$ is particularly striking if one selects stars according to a metallicity dependent limit in $T_{\text {eff }}$ (see Fig. 3 in Meléndez et al. 2010). Apparently, the scatter in $A(\mathrm{Li})$ below $[\mathrm{Fe} / \mathrm{H}] \simeq-2.5$, as found in some of the works cited above, is due to an increased degree of lithium depletion in the lower-mass stars.

The Spite plateau was originally interpreted as evidence of the primordial lithium abundance and used to constrain the baryon density in the Big Bang from nucleosynthesis calculations. Adopting the WMAP value of the cosmic baryon density (Komatsu et al. 2011), standard Big Bang nucleosynthesis predicts, however, a primordial lithium abundance, $A\left({ }^{7} \mathrm{Li}\right)=$ $2.72 \pm 0.06$ (Cyburt et al. 2008; Coc et al. 2012), i.e. about a factor of three higher than the abundance corresponding to the Li-plateau.

A possible explanation of this so-called "lithium problem" could be that lithium in the atmospheres of stars on the Spite plateau is depleted by gravitational settling and/or by mixing with gas from layers with such a high temperature $\left(T \gtrsim 2.5 \times 10^{6} \mathrm{~K}\right)$ that $\mathrm{Li}$ is destroyed by reactions with protons. Stellar models including rotational induced mixing (Pinsonneault et al. 1999; Piau 2008), atomic diffusion (Salaris \& Weiss 2001; Richard et al. 2002) and internal gravity waves (Talon \& Charbonnel 2004) predict a significant Li depletion, but they have difficulties in explaining a depletion of $0.5 \mathrm{dex}$ and a Li-plateau with very little scatter in $A(\mathrm{Li})$. The models of Richard et al. (2005), which include atomic and turbulent diffusion, are more successful, when a free parameter describing the degree of turbulent mixing is optimized. It remains, however, to be seen if such models can explain the decline in $A(\mathrm{Li})$ for $[\mathrm{Fe} / \mathrm{H}] \lessgtr-2.5$; Richard et al. argue in fact that Li depletion in stars on the main sequence does not depend on metallicity.

In addition to "in situ" stellar depletion of lithium, Piau et al. (2006) suggest that the $\mathrm{Li}$ abundance of gas in the Galactic halo may have been reduced by nuclear burning in massive, zero-metallicity (Population III) stars before low-mass stars on the Spite plateau formed. The decline and/or increased scatter of $A(\mathrm{Li})$ at low metallicity, could then be due to incomplete mixing of gas and preferential star formation in the vicinity of supernovae when $[\mathrm{Fe} / \mathrm{H}] \lesssim-2.5$. In order to obtain a significant reduction of the primordial lithium abundance, say $0.3 \mathrm{dex}$, about $50 \%$ of the gas in the early halo has to pass through Pop III stars. If these stars have masses in the range 10-40 $M_{\odot}$ and current nucleosynthesis models are correct, they would, however, produce an excessive amount of $\mathrm{CNO}$ elements compared to the observed abundances in metal-poor halo stars (Prantzos 2007). Very massive stars collapsing as black holes would be better candidates provided that they eject their Li-depleted envelope, but not the metal-rich core.

A more radical solution of the lithium problem may be found if decaying supersymmetric particles play a role in Big Bang nucleosynthesis (BBN). For the right combination of the properties of such putative particles, the abundance of lithium can be reduced to a level that agrees with $A(\mathrm{Li})$ in stars on the Spite plateau (see review by Fields 2011) without spoiling the agreement between the deuterium abundance calculated from standard BBN and that measured in quasar absorption line systems (Pettini et al. 2008). However, non-standard BBN cannot explain the dependence of $A(\mathrm{Li})$ on metallicity below $[\mathrm{Fe} / \mathrm{H}] \sim-2.5$, so some stellar depletion or Galactic astration of lithium must be present.
It is clear from this discussion that the lithium problem is far from being solved and that new observations and further theoretical works in the fields of stellar physics, Galactic evolution and primordial nucleosynthesis are needed. Regarding new observational data, we note that recent efforts have concentrated on lithium abundances in very metal-poor stars, whereas the metalrich end of the Spite plateau has been somewhat neglected. In the present paper, we try to remedy this by making a study of $\mathrm{Li}$ abundances in a sample of halo and thick-disk main-sequence stars with $-1.6<[\mathrm{Fe} / \mathrm{H}]<-0.4$ for which we have previously determined precise abundances of a number of elements from $\mathrm{Na}$ to Ba (Nissen \& Schuster 2010, 2011, hereafter Papers I and II). These abundances have revealed the existence of two distinct halo populations in the solar neighborhood with a clear separation in $[\alpha / \mathrm{Fe}]^{1},[\mathrm{Na} / \mathrm{Fe}],[\mathrm{Ni} / \mathrm{Fe}],[\mathrm{Cu} / \mathrm{Fe}]$, and $[\mathrm{Zn} / \mathrm{Fe}]$. The kinematics and ages (Schuster et al. 2012, Paper III) of the stars suggest that the high-alpha halo stars were formed in situ in the inner regions of the Galaxy, whereas the low-alpha stars were accreted from satellite galaxies. Hence, we have a unique possibility to see if lithium abundances of stars formed in different environments are the same, which is of particular interest in connection with the suggestion of Piau et al. (2006) of lithium destruction in an early generation of massive stars. Furthermore, the sample of stars allows us to investigate trends of $A(\mathrm{Li})$ as a function of stellar mass and heavy-element abundance, which may be used to constrain theories of lithium depletion in stars.

In Sect. 2, we describe how the lithium abundance $A(\mathrm{Li})$, stellar mass $M$, and total heavy-element mass fraction $Z$ are determined and check the errors of these parameters by comparing with Meléndez et al. (2010) for 24 stars in common. In Sect. 3, the dependence of $A(\mathrm{Li})$ on $M$ and $Z$ is investigated. In Sect. 5, the results are discussed and some concluding remarks are given in Sect. 6.

\section{Determination of $A(\mathrm{Li}), M$, and $Z$}

\subsection{Lithium abundances}

The abundance of $\mathrm{Li}$ is determined from the equivalent width (EW) of the Li I 16707.8 resonance line as measured in highresolution spectra obtained with UVES at the ESO/VLT and FIES at the Nordic Optical Telescope. For a more detailed description of these spectra we refer to Paper I. The signal-to-noise ratio $(S / N)$ of the UVES spectra is very high, i.e. ranging from about 200 to 600 , but some of the UVES spectra suffer from a small residual fringing at the wavelength of the $\mathrm{Li}$ I line. The FIES spectra have lower $S / N$ (140 to 200) but are free from fringing. The typical 1-sigma error of an EW measurement is estimated to be $\pm 1 \mathrm{~m} \AA$. This is confirmed by comparing data for six stars observed with both UVES and FIES (see Paper I, Table 2). The mean difference (FIES - UVES) is $0.2 \mathrm{~m} \AA$ with a rms deviation of $1.1 \mathrm{~m} \AA$. There is also very good agreement with Meléndez et al. (2010); for 24 stars in common, the mean EW difference is $0.02 \mathrm{~m} \AA$ and the rms deviation is $1.0 \mathrm{~m} \AA$.

The measured equivalent widths are given in Table 1. For 25 stars, the Li I line could not be detected. Instead, two-sigma upper limits, as estimated from the $S / N$ of the spectrum, are given. Furthermore, one star in Paper I (HD 17820) is excluded, because the Li I line is strongly disturbed by a cosmic ray hit.

For each star a model atmosphere has been obtained from the MARCS grid (Gustafsson et al. 2008) by interpolating to the $T_{\text {eff }}, \log g,[\mathrm{Fe} / \mathrm{H}]$, and $[\alpha / \mathrm{Fe}]$ values of the star. The Uppsala

\footnotetext{
${ }^{1} \alpha$ refers to the average abundance of $\mathrm{Mg}, \mathrm{Si}, \mathrm{Ca}$, and $\mathrm{Ti}$.
} 
program BSYN is used to calculate the profile and equivalent width of the $\mathrm{Li}$ I $\lambda 6707.8$ line as a function of $A(\mathrm{Li})$ assuming local thermodynamic equilibrium (LTE). Interpolation to the observed EW-value then yields $A(\mathrm{Li})$.

The wavelengths and $g f$-values of the hyperfine components of the Li I $\lambda 6707.8$ line are adopted from Sansonetti et al. (1995) and Yan \& Drake (1995); see Smith et al. (1998, Table 3). ${ }^{6} \mathrm{Li}$ components are not included. If the isotopic ${ }^{6} \mathrm{Li} /{ }^{7} \mathrm{Li}$ ratio is on the order of 5\%, as found by Asplund et al. (2006) for some stars, the profile of the Li line will be slightly broader than calculated, but the EW is practically the same for a given total lithium abundance, because the Li line is far from being saturated. In practice, we are therefore determining the total lithium abundance. With the resolutions of the spectrographs applied, $R=60000$ for UVES and $R=40000$ for FIES, it is not possible to detect ${ }^{6} \mathrm{Li}$ at a level of $5 \%$, but from the relation between the center-of-gravity ( $\mathrm{cog}$ ) wavelength of the Li I line and the ${ }^{6} \mathrm{Li}$ fraction (Smith et al. 1998, Eq. (1)), the measured cog-wavelengths indicate that the ${ }^{6} \mathrm{Li} /{ }^{7} \mathrm{Li}$ ratio is less than about $10 \%$.

Effective temperatures and surface gravities are determined spectroscopically, i.e. $T_{\text {eff }}$ from the excitation balance of weak $\mathrm{Fe}$ I lines and $\log g$ from the ratio of iron abundances derived from $\mathrm{Fe}_{\mathrm{I}}$ and $\mathrm{Fe}$ II lines. As described in Paper I, the zero points for these parameters are set by two nearby, unreddened "standard" stars, HD 22879 and HD 76932. Their effective temperatures were determined from the color indices $(b-y)$ and $(V-K)$ using the calibrations of Ramírez \& Meléndez (2005). The recent more accurate calibrations by Casagrande et al. (2010) shows, however, a systematic offset of about $+100 \mathrm{~K}$ in $T_{\text {eff }}$ relative to the Ramírez \& Meléndez values for stars with $[\mathrm{Fe} / \mathrm{H}]>$ -2.0 and $4800<T_{\text {eff }}<6200 \mathrm{~K}$. We have, therefore, increased the $T_{\text {eff }}$ values given in Paper I by $100 \mathrm{~K}$. As discussed in Paper III, this leads to a systematic correction of +0.03 dex of $\log g$ and corrections of $[\mathrm{Fe} / \mathrm{H}]$ ranging from about $-0.03 \mathrm{dex}$ at $T_{\text {eff }}=5400 \mathrm{~K}$ to $+0.01 \mathrm{dex}$ at $T_{\text {eff }}=6100 \mathrm{~K}$, whereas the correction of $[\alpha / \mathrm{Fe}]$ is approximately constant at $-0.01 \mathrm{dex}$. The $+100 \mathrm{~K}$ correction of $T_{\text {eff }}$ changes the derived lithium abundances by about +0.08 dex, whereas the corresponding changes of $\log g,[\mathrm{Fe} / \mathrm{H}]$, and $[\alpha / \mathrm{Fe}]$ have no significant effect on $A(\mathrm{Li})$. The values of $\log g,[\mathrm{Fe} / \mathrm{H}]$, and $[\alpha / \mathrm{Fe}]$ given in Paper I have, therefore, been adopted.

The derived LTE lithium abundances are given in Table 1 together with the adopted model atmosphere parameters, $T_{\text {eff }}$, $\log g,[\mathrm{Fe} / \mathrm{H}]$, and $[\alpha / \mathrm{Fe}]$. The table also lists non-LTE lithium abundances based on the NLTE - LTE corrections calculated by Lind et al. (2009) for 1D MARCS models and collisional cross sections from Barklem et al. (2003). These corrections depend mainly on the effective temperature and range from about $+0.04 \mathrm{dex}$ at $T_{\mathrm{eff}}=5400 \mathrm{~K}$ to $-0.06 \mathrm{dex}$ at $T_{\mathrm{eff}}=6100 \mathrm{~K}$. 3D non-LTE calculations are not available for the metallicities of our stars, but for a sample of very metal-poor stars Sbordone et al. (2010) find only small differences in $A(\mathrm{Li})( \pm 0.02 \mathrm{dex})$, when 1D LTE and 3D NLTE analyses are compared.

The error of $A(\mathrm{Li})$ is mainly caused by the error of $T_{\text {eff. }}$ In Paper III, the one-sigma error of the differential $T_{\text {eff }}$ values was estimated to be on the order of $\pm 30 \mathrm{~K}$ from a comparison of our spectroscopic temperatures with values determined from $(b-y)$ and $(V-K)$ for a sample 44 stars that are unreddened according to the absence of interstellar Na I D lines. The corresponding error of $A(\mathrm{Li})$ is 0.025 dex. Adding the small error arising from the EW measurements, we arrive at a statistical error of $\pm 0.03 \mathrm{dex}$ in $A(\mathrm{Li})$ if the equivalent width of the $\mathrm{Li}$ I $\lambda 6707.8$ line is greater than about $20 \mathrm{~m} \AA$.

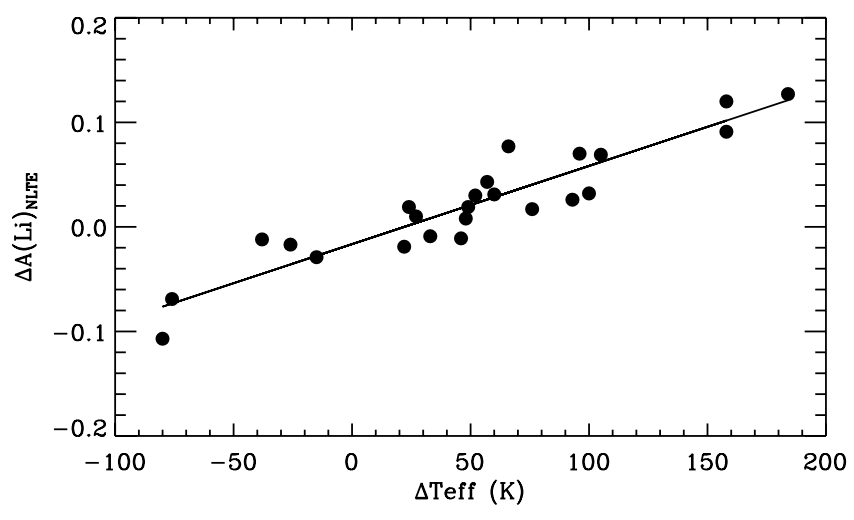

Fig. 1. Differences between Li abundances in Meléndez et al. (in prep.) and in this paper versus the corresponding differences in $T_{\text {eff }}$.

To check the accuracy of our Li abundances, we have compared with Meléndez et al. (2010) for 24 stars in common using a slightly revised version of their Table 1 (Meléndez et al., in prep.). MARCS models are also used to derive their abundances, but $T_{\text {eff }}$ is determined with the infrared flux method (IRFM) as implemented by Casagrande et al. (2010) and with the use of interstellar $\mathrm{Na}$ I $\mathrm{D}$ lines to estimate the reddening. The mean difference (Meléndez - this paper) is $\langle\Delta A(\mathrm{Li})\rangle=0.022$ with a scatter of $\sigma=0.054 \mathrm{dex}$, which corresponds quite well to the estimated errors of $A(\mathrm{Li})$, i.e. \pm 0.035 in Meléndez et al. (2010) and \pm 0.030 dex in this paper.

As seen from Fig. 1, the differences in $A(\mathrm{Li})$ are closely correlated with the corresponding differences in $T_{\text {eff }}$; the scatter around the regression line $\left(\Delta A(\mathrm{Li})=-0.016+0.00075 \Delta T_{\text {eff }}\right)$ is only $0.021 \mathrm{dex}$. This shows that the differences in $A(\mathrm{Li})$ between Meléndez et al. and this paper are mainly caused by differences in $T_{\text {eff }}$ with only a small contribution coming from the differences in the measured equivalent widths of the $\mathrm{Li}$ I $\lambda 6707.8$ line.

The mean deviation in effective temperature (Meléndez this paper) is $\left\langle\Delta T_{\text {eff }}\right\rangle=51 \mathrm{~K}$ with $\sigma=66 \mathrm{~K}$. A comparison with Casagrande et al. (2010) for 43 stars in common results, however, in a smaller mean difference (Casagrande - this paper) $\left\langle\Delta T_{\text {eff }}\right\rangle=16 \mathrm{~K}$ with $\sigma=61 \mathrm{~K}$. Furthermore, a comparison with the IRFM temperatures of González Hernández \& Bonifacio (2009) for 45 stars in common gives a mean difference (Gonzalez - this paper) $\left\langle\Delta T_{\text {eff }}\right\rangle=-16 \mathrm{~K}$ with $\sigma=81 \mathrm{~K}$. Altogether, we estimate that the uncertainty of the $T_{\text {eff }}$-scale is on the order of $\pm 50 \mathrm{~K}$. The corresponding error of $A(\mathrm{Li})$ is about 0.04 dex.

\subsection{Stellar mass and heavy-element fraction}

Stellar masses have been determined from the $\mathrm{Y}^{2}$ (Yonsei-Yale) evolutionary tracks published by Yi et al. (2003). Their stellar models include convective core overshoot and helium diffusion, and tracks are available for a set of total heavy-element mass fractions, ranging from $Z=0.00001$ to $Z=0.08$. The helium mass fraction is given by $Y=0.23+2.0 \mathrm{Z}$, and the tracks are computed for $[\alpha / \mathrm{Fe}]=0.0,0.3$ and 0.6. Oxygen is considered as an alpha-element, whereas carbon and nitrogen are assumed to follow iron.

For a given value of $Z$, the three available $[\mathrm{Fe} / \mathrm{H}]-[\alpha / \mathrm{Fe}]$ combinations lead to practically the same mass tracks. For each star, we have therefore first calculated $Z_{\text {star }}$ from the measured $[\mathrm{Fe} / \mathrm{H}]$ and $[\alpha / \mathrm{Fe}]$ values and then determined two masses by interpolating between tracks in the $\log g-\log T_{\text {eff }}$ plane for the two adjacent $Z$-values available. Interpolation to $Z_{\text {star }}$ then leads 


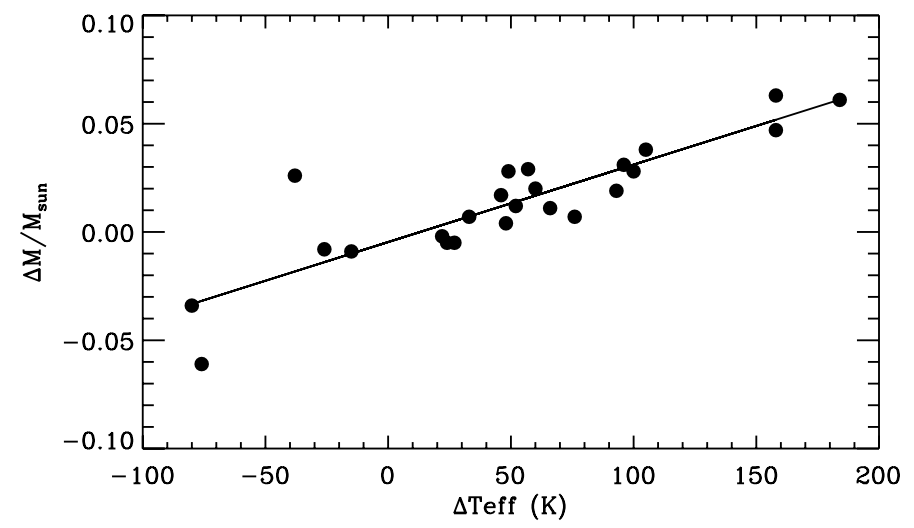

Fig. 2. Differences between stellar masses in Meléndez et al. (in prep.) and in this paper versus the corresponding differences in $T_{\text {eff }}$.

to the mass of the star. The conversion from $[\mathrm{Fe} / \mathrm{H}]$ and $[\alpha / \mathrm{Fe}]$ to $Z_{\text {star }}$ followed the procedure adopted for the $\mathrm{Y}^{2}$ tracks and isochrones (Kim et al. 2002, Table 2). It is based on a solar abundance distribution (Grevesse \& Noels 1993) corresponding to $Z_{\odot}=0.0181$, whereas the more recent $3 \mathrm{D}$, non-LTE solar abundance distribution determined by Asplund et al. (2009) corresponds to $Z_{\odot}=0.0134$. If this smaller value of the solar heavy-element fraction had been adopted, the stellar $Z$ values would decrease by $25 \%$, and the derived masses would change somewhat, although we expect the effect on the relative masses to be small. When stellar evolutionary tracks based on the Asplund et al. (2009) abundance distribution become available, improved masses can be derived. The recent Geneva model grid (Mowlavi et al. 2012) is based on this distribution, but is limited to $Z$ values from 0.006 to 0.04 , an interval which covers only a small fraction of our stars.

As discussed in Paper III, there is a small systematic offset in $\log g$ between the $\mathrm{Y}^{2}$ isochrones and our nearly unevolved $\left(T_{\text {eff }}<5600 \mathrm{~K}\right)$ main-sequence stars. The reason for this is unclear, but could be related to a change of the mixing length parameter as a function of metallicity. To avoid this mismatch we have introduced a correction of -0.13 dex to the $\log g$ values of the $\mathrm{Y}^{2}$ models before using the evolutionary tracks to determine stellar masses.

The derived masses in units of the solar mass are given in Table 1 together with $Z$ values derived from $[\mathrm{Fe} / \mathrm{H}]$ and $[\alpha / \mathrm{Fe}]$. Based on the statistical one-sigma errors given in Paper I, $\sigma\left(T_{\text {eff }}\right)=30 \mathrm{~K}, \sigma(\log g)=0.05 \operatorname{dex}, \sigma([\mathrm{Fe} / \mathrm{H}])=0.03$ dex, and $\sigma([\alpha / \mathrm{Fe}])=0.02$ dex, we estimate that the statistical error of the mass is $\sigma\left(M / M_{\odot}\right) \simeq 0.02$. The systematic error is on the same order of size; an offset of $+50 \mathrm{~K}$ in the $T_{\text {eff }}$-scale would lead to a systematic change of +0.018 in $M / M_{\odot}$.

Meléndez et al. (2010) also derived stellar masses from the $\mathrm{Y}^{2}$ models using both $\log g$ and absolute magnitudes (determined from Hipparcos parallaxes) in combination with $\log T_{\text {eff }}$. $[\alpha / \mathrm{Fe}]$ is assumed to be +0.3 in all stars with $[\mathrm{Fe} / \mathrm{H}]<-1.0$, which is close to the average value $(+0.27)$ for the 24 stars in common with our study. The mean mass difference (Meléndez - this paper) is $\left\langle M \mid M_{\odot}\right\rangle=0.014$ with $\sigma=0.028$, which supports a statistical error for $M / M_{\odot}$ on the order of \pm 0.02 in both works. As seen from Fig. 2, the differences between the two sets of derived masses are closely correlated with the corresponding differences in $T_{\text {eff. }}$ The scatter around the regression line $\left(\Delta M / M_{\odot}=-0.0047+0.00036 \Delta T_{\text {eff }}\right)$ is only $\pm 0.014 M / M_{\odot}$.

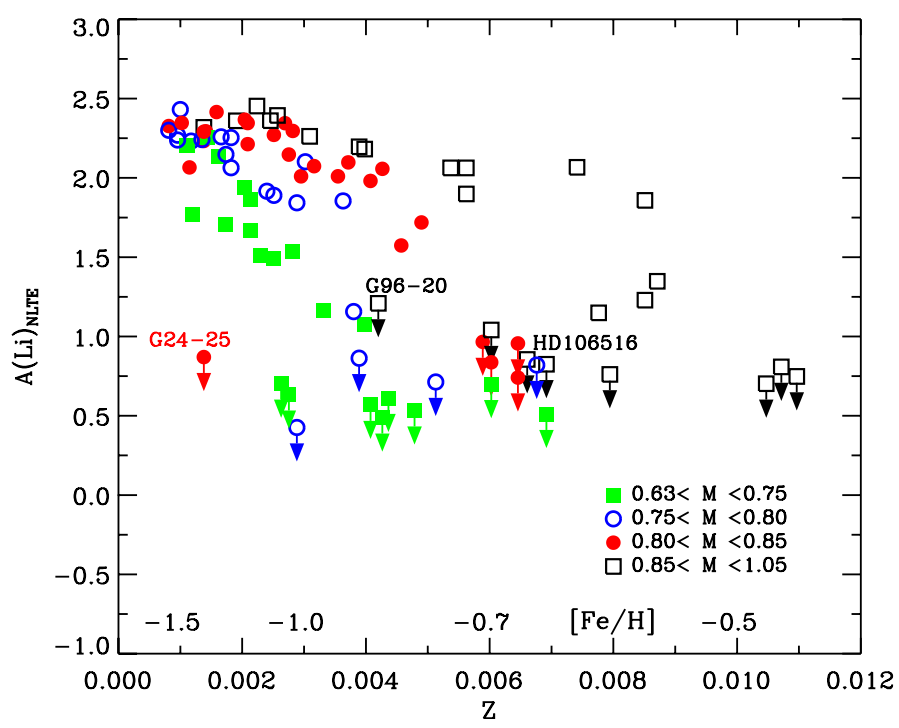

Fig. 3. Li abundances as a function of the heavy-element mass fraction, $Z$, for all stars in Table 1. Upper limits of $A(\mathrm{Li})$ are indicated with a downward directed arrow. Approximate $[\mathrm{Fe} / \mathrm{H}]$-values are shown above the $x$-axis.

\section{Lithium versus mass and heavy-element fraction}

In Fig. 3, the Li abundances for all stars in Table 1 are plotted against the heavy-element mass fraction with different symbols for the four mass ranges indicated. Disregarding 25 stars with an upper limit for the $\mathrm{Li}$ abundance, it is seen that $A(\mathrm{Li})$ depends in a systematic way on mass and $Z$. $A(\mathrm{Li})$ decreases when $Z$ increases, and for a given $Z$-value, $A(\mathrm{Li})$ decreases with decreasing mass. Corresponding relations have been obtained by Ryan \& Deliyannis (1998) and Boesgaard et al. (2005), who used $T_{\text {eff }}$ for main-sequence stars as a substitute for mass, and by Meléndez et al. (2010), who investigated $A(\mathrm{Li})$ as a function of mass for selected metallicity groups.

Figure 4 shows in more detail how the measured lithium abundances depend on $Z$ for the four mass ranges indicated. Stars for which the Li line could not be detected are excluded from this figure. For a given mass, $A(\mathrm{Li})$ varies approximately linearly with $Z$ but the slope becomes steeper with decreasing stellar mass. As seen from Fig. 5, the dependence of $A(\mathrm{Li})$ on mass is also close to linear for a given $Z$-value. This suggests that $A(\mathrm{Li})$ can be fitted with a function

$A(\mathrm{Li})_{\mathrm{fit}}=a_{0}+a_{1} M+a_{2} Z+a_{3} M Z$,

where $a_{0}, a_{1}, a_{2}$, and $a_{3}$ are constants. Taking into account the estimated errors, $\sigma(A(\mathrm{Li}))=0.03, \sigma\left(M / M_{\odot}\right)=0.02$, and $\sigma(\log Z)=0.04$, chi-square minimization leads to the expression

$A(\mathrm{Li})_{\mathrm{fit}}=2.253+0.405 M-2043 Z+2280 M Z$,

where $M$ is in units of the solar mass, and NLTE Li abundances have been applied. This fit, which is obtained for 59 stars having $0.7<M / M_{\odot}<0.90$ and $Z<0.006$, has a reduced chi-square of $\chi_{\text {red }}^{2}=1.12$. One star, G 16-20, within these ranges is excluded, because it has a 5.5-sigma deviation in $A(\mathrm{Li})$. If this star is included in the fit, the chi-square rises to an unacceptably high value of $\chi_{\text {red }}^{2}=1.71$. G 16-20 is the most evolved star in the sample $(\log g=3.64)$, so lithium may have been diluted due to the deepening of the convection zone as the star evolved up along the subgiant branch. 
P. E. Nissen and W. J. Schuster: Lithium abundances in two distinct halo populations

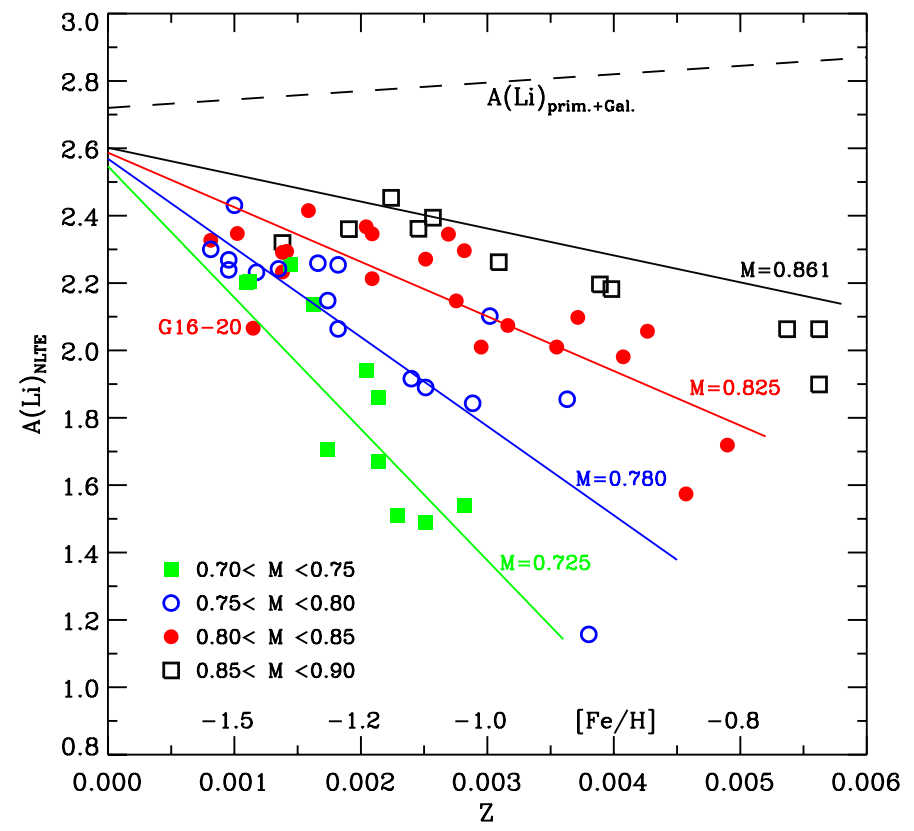

Fig. 4. $\mathrm{Li}$ abundances as a function of the heavy-element fraction for stars with $0.70<M / M_{\odot}<0.90$ and $Z<0.006$. For each of the four mass intervals, the fit given in Eq. (2) is shown as a straight line corresponding to the mean mass of the group indicated at the line. The dashed line shows the sum of the primordial Li abundance predicted from standard $\mathrm{BBN}$ calculations and ${ }^{7} \mathrm{Li}$ made in the Galaxy due to cosmic ray and stellar nucleosynthesis reactions according to Prantzos (2007).

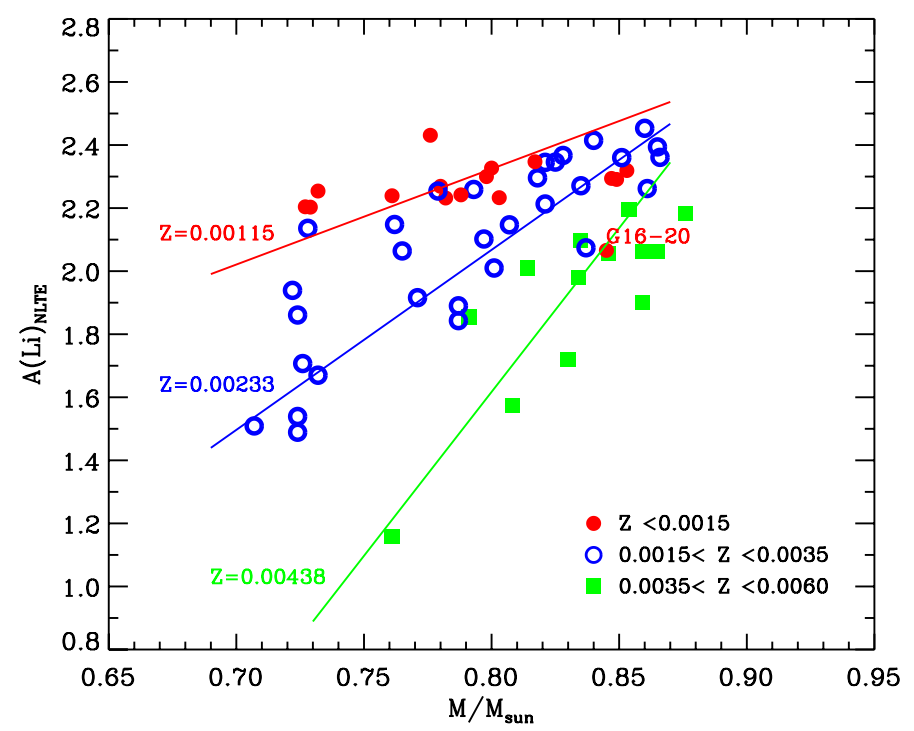

Fig. 5. Li abundances as a function of stellar mass for stars with $0.70<$ $M / M_{\odot}<0.90$ and $Z<0.006$. For each of the three $Z$-intervals, the fit given in Eq. (2) is shown as a straight line corresponding to the mean value of $Z$ for the group indicated at the line.

The fit in Eq. (2) is shown as straight lines in Fig. 4 for the mean values of $M$ for each of the four mass intervals, and in Fig. 5 for the mean values of $Z$ of the three metallicity groups. Some of the dispersion around these lines is due to the ranges in $M$ and $Z$, respectively, for each group. The quality of the fit in Eq. (2) can be better seen from Fig. 6, where the residuals are shown as a function of the heavy-element fraction with individual error bars given. As seen, the error of $\Delta=A(\mathrm{Li})-A(\mathrm{Li})_{\mathrm{fit}}$

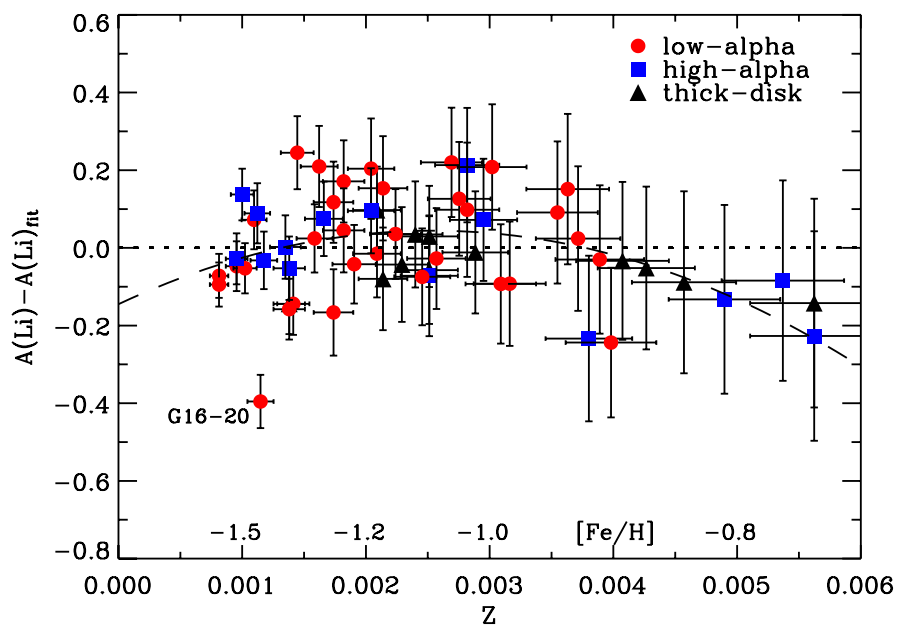

Fig. 6. The residuals of the fit given in Eq. (2) as a function of the heavyelement fraction, $Z$. One-sigma error bars are shown. The dashed line shows a parabolic fit to the residuals.

increases with increasing $Z$, which is related to the fact that both the mass-induced error

$\sigma(\Delta)_{M}=\left(a_{1}+a_{3} Z\right) \sigma(M)$

and the $Z$-induced error

$\sigma(\Delta)_{Z}=\left(a_{2}+a_{3} M\right) \sigma(Z)=\left(a_{2}+a_{3} M\right) \ln 10 Z \sigma(\log Z)$

increase with $Z$.

As seen from Fig. 6, stars with $0.0015<Z<0.003$ tend to have positive residuals from the fit in Eq. (2), whereas stars with $Z>0.004$ have negative residuals. This suggests that there is a small curvature in the relation between $A(\mathrm{Li})$ and $Z$, which is not taken care of in the fit. If the residuals are fitted with a parabola as shown with the dashed line in Fig. 6, the reduced chi-square decreases slightly, i.e. from $\chi_{\text {red }}^{2}=1.12$ to 1.03 .

Some of the stars with a non-detectable $16707.8 \mathrm{Li}$ I line have an upper limit of $A(\mathrm{Li})$ much below the value predicted from Eq. (2). In a few cases we may identify the reason for the low lithium abundance. Three such stars are marked in Fig. 3. G 96-20 and HD 106516 have masses around one solar mass, and may be blue stragglers; they are significantly bluer than the turnoff of halo and thick-disk stars with similar metallicities (Carney et al. 2005). Thus, their low lithium abundance could be due to merging with a companion star causing Li-depleted gas to be mixed into the stellar atmosphere. The third star, G 24-25, is an $s$-process rich star probably due to mass transfer from a former AGB component (Liu et al. 2012), which also brings Li-depleted gas into the atmosphere. HD 106516 and G 24-25 are singlelined spectroscopic binaries (Latham et al. 2002; Ducati et al. 2011) supporting the mass transfer hypothesis as an explanation of the abnormally low lithium abundance.

As noted in the last column of Table 1 several other stars are single-lined spectroscopic binaries, and five stars are visual binaries according to the HIPPARcos catalogue (ESA 1997) with components so close (within about one arcsec) that the component may have affected the observed spectrum. None of these binary stars stand out with respect to the lithium abundance. HD 219617 is a particularly interesting case. According to Takeda \& TakadaHidai (2011), the two components have nearly the same magnitudes $(V=8.77$ and 9.08$)$ and are separated by 0.8 arcsec. The VLT/UVES spectrum of this star was obtained with a 0.8 arcsec slit under rather poor seeing conditions, and is thus an unspecified average of the two spectra. 


\section{Discussion}

According to standard models of $\mathrm{F}$ and $\mathrm{G}$ main-sequence stars (e.g. Deliyannis et al. 1990), the depth and thus the temperature at the bottom of the upper convection zone increase when the stellar mass decreases. Hence, the destruction of Li by reactions with protons becomes more effective as the mass decreases, which explains the dependence of $A(\mathrm{Li})$ on stellar mass seen in Fig. 5. Models including mixing and diffusion (Pinsonneault et al. 1999; Salaris \& Weiss 2001; Richard et al. 2005) also predict a mass dependence of $A(\mathrm{Li})$ qualitatively similar to what is seen in Fig. 5. It remains, however, to be seen if these models can also reproduce the observed dependence of $A(\mathrm{Li})$ on $Z$ for a given mass (Fig. 4). In any case, the trend of $A(\mathrm{Li})$ as a function of mass and $Z$ sets important constraints on stellar modelling.

It should be emphasized that the relation between $A(\mathrm{Li}), M$, and $Z$ given in Eq. (2) is only valid for main-sequence stars with $0.7<M / M_{\odot}<0.9$ and $0.001 \lesssim Z<0.006$ and with a lithium line detected. For stars more metal-rich than $Z=0.006^{2}, A(\mathrm{Li})$ shows a large scatter at a given mass and $Z$, which can be seen from Fig. 3. Obviously, other parameters than $M$ and $Z$ affect the depletion of lithium for these more metal-rich stars. A similar result has been obtained for $\mathrm{F}$ and $\mathrm{G}$ main-sequence stars belonging to the Galactic disk by Chen et al. (2001) and Lambert \& Reddy (2004), who discuss if age and/or initial angular momentum play a role. The disk stars span a large range in age, i.e. they have had different times to deplete lithium, and the recent work by Baumann et al. (2010) on lithium abundances of solar twins shows that $A(\mathrm{Li})$ depends quite steeply on age. The halo and thick-disk stars in our sample have, however, rather uniform ages, i.e. 10-13 Gyr as shown in Paper III, and it seems unlikely that differences in age could be the reason of the large scatter in $A(\mathrm{Li})$ for stars with $0.006<Z<0.012$.

As seen from Fig. 4, the value of $A(\mathrm{Li})_{Z=0}$, obtained by extrapolating Eq. (2) to $Z=0$ for a given mass, is nearly independent of mass. For the mean mass of the sample, $\langle M\rangle=0.80 M_{\odot}$, we obtain $A(\mathrm{Li})_{Z=0}=2.58 \pm 0.04$, where the (1-sigma) error is determined from the chi-square analysis used to derive Eq. (2). The uncertainty of the $T_{\text {eff }}$-scale $( \pm 50 \mathrm{~K})$ introduces an additional systematic uncertainty of \pm 0.04 dex. Had we, instead, adopted the parabolic fit shown in Fig. 6, $A(\mathrm{Li})_{Z=0}$ would be lowered by 0.14 dex, but this gives too much weight to the high- $Z$ stars in the extrapolation. An alternative approach is to limit the linear fit to stars with $Z<0.003$, which results in a decrease of $A(\mathrm{Li})_{Z=0}$ by 0.08 dex relative to the value obtained when using Eq. (2) for the extrapolation. On the other hand, if the fit is based on the LTE lithium abundances, which would be more in line with the small 3D, non-LTE corrections of Sbordone et al. (2010), $A(\mathrm{Li})_{Z=0}$ increases by approximately 0.08 dex. Altogether, we estimate

$A(\mathrm{Li})_{Z=0}=2.58 \pm 0.04_{\text {stat }} \pm 0.10_{\text {syst }}$.

Considering the uncertainties, this value is in reasonable agreement with the primordial ${ }^{7} \mathrm{Li}$ abundance

$A\left({ }^{7} \mathrm{Li}\right)_{\text {prim }}=2.72 \pm 0.06$

predicted from standard BBN calculations (Cyburt et al. 2008; Coc et al. 2012) and the WMAP baryon density (Komatsu et al. 2011).

The derived lithium abundances, therefore, seem compatible with a scenario where the metal-rich halo stars were formed out

\footnotetext{
2 The limit $Z<0.006$ corresponds to $[\mathrm{Fe} / \mathrm{H}] \lesssim-0.7$ for high-alpha stars and $[\mathrm{Fe} / \mathrm{H}] \lessgtr-0.6$ for low-alpha stars.
}

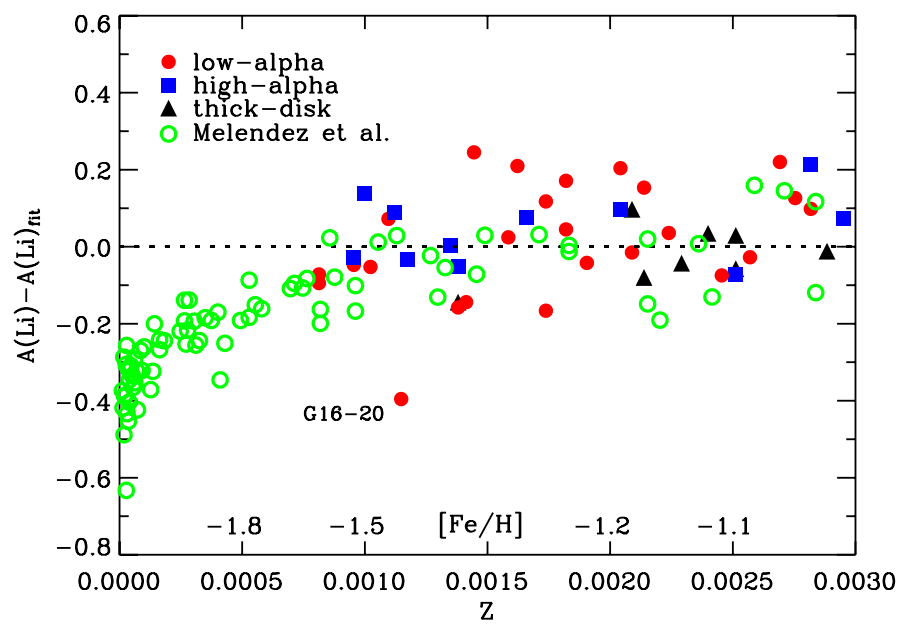

Fig. 7. The residuals in Eq. (2) as a function of $Z$ with stars from Meléndez et al. (in prep.) included.

of gas having a lithium abundance equal to that predicted from standard BBN plus a contribution from Galactic processes followed by depletion of lithium in the stellar atmospheres depending approximately linearly on mass and $Z$. The Galactic evolution of ${ }^{7} \mathrm{Li}$, due to cosmic ray processes (i.e. $p+C N O$ and $\alpha+\alpha$ ) and $\mathrm{Li}$ production in AGB stars and novae, has been modelled by Prantzos (2007) and is shown as a dashed line in Fig. 4. As seen, the Galactic contribution to ${ }^{7} \mathrm{Li}$ is small compared to the primordial value and increases linearly with $Z$. There is also a small contribution to ${ }^{6} \mathrm{Li}$ from cosmic ray processes but since this isotope is much more fragile to proton reactions than ${ }^{7} \mathrm{Li}$, the abundance of ${ }^{6} \mathrm{Li}$ will be negligible, even for a small degree of ${ }^{7} \mathrm{Li}$ destruction.

By extension of this scenario to more metal-poor halo stars, we would expect that the difference between the lithium abundance measured for stars on the Spite plateau and that predicted from standard BBN and the WMAP baryon density is also due to $\mathrm{Li}$ depletion in the stars. The simple linear relation between $A(\mathrm{Li}), M$, and $Z$ derived for stars with $0.001 \lesssim Z<0.006$ is, however, not valid for more metal-poor stars. This can be seen from Fig. 7, where we have included stars from Meléndez et al. (in prep.). For $Z \gtrsim 0.001$, the Meléndez et al. stars agree well with our fit, but at lower metallicities, the Li abundances fall below the values expected from Eq. (2). On the Spite plateau $(0.0001 \lesssim Z \lesssim 0.001), A(\mathrm{Li})-A(\mathrm{Li})_{\text {fit }}$ is nearly independent of mass and $Z$, but at the lowest metallicities $(Z \lesssim 0.0001)$ $A(\mathrm{Li})-A(\mathrm{Li})_{\text {fit }}$ varies among the stars. Interestingly, the work of Meléndez et al. (2010) suggests that this scatter is related to differences in stellar masses.

As seen from Fig. 6, the distribution of the residuals of the fit given in Eq. (2) is nearly the same for high- and low-alpha halo stars. Given that the high-alpha stars are likely to have been formed in situ in the inner part of the Galaxy, whereas the lowalpha ones were accreted from satellite galaxies (Purcell et al. 2010; Zolotov et al. 2009, 2010), this means that stars belonging to such different systems were formed with the same Li abundance, and later depleted lithium in the same way as a function of mass and $Z$. This result is difficult to reconcile with the proposal by Piau et al. (2006) of astration of lithium in an early generation of massive, zero-metallicity Population III stars. It would require that the same mass fraction of interstellar gas has been processed by massive stars in the inner part of the Galaxy and in satellite galaxies. 
The similarity of Li abundances in high- and low-alpha halo stars may seem surprising in view of the recent study of beryllium by Tan $\&$ Zhao (2011). At a given $[\alpha / \mathrm{H}]$ abundance, they find the low-alpha stars to be systematically underabundant in Be relative to the high-alpha stars. The difference in $A(B e)$ is about 0.2 dex. Since Be is produced by cosmic ray processes, i.e. $\mathrm{CNO}$ nuclei impinging on interstellar $\mathrm{H}$ and $\mathrm{He}$, the explanation of the lower Be abundance in low-alpha stars may be that the cosmic ray nucleosynthesis is less efficient in dwarf satellite galaxies than in the inner part of the Galaxy (Prantzos 2012). This would also lead to a lower cosmic ray production of lithium, but since the Galactic part of the initial stellar abundance of lithium is small compared to the primordial part, it will have only a marginal effect on the measured stellar lithium abundances.

According to Paper III, the high-alpha halo stars have ages 2-3 Gyr larger than the low-alpha ones. Thus, the high-alpha stars have had more time to deplete lithium than low-alpha stars. As we see no significant difference in their $\mathrm{Li}$ abundances, this suggests that Li depletion mainly occurred in an early stage of the stellar evolution, i.e. either in the pre-main-sequence phase or near the zero-age main sequence before the star evolved up towards the turn-off region.

\section{Concluding remarks}

In this paper, we have made a precise, homogeneous study of lithium abundances in a sample of main-sequence stars for which a previous study (Paper I) has revealed the existence of two distinct halo populations with different trends of $[\alpha / \mathrm{Fe}]$ for the metallicity range $-1.4<[\mathrm{Fe} / \mathrm{H}]<-0.7$. The kinematics of the stars and Galactic formation models suggest that the low-alpha stars have been accreted from satellite galaxies, including the progenitor of $\omega \mathrm{Cen}$, whereas the high-alpha stars were formed in situ in the disk or bulge and later displaced to the halo by the merging dwarf galaxies (Purcell et al. 2010; Zolotov et al. 2009, 2010).

Precise values of the heavy-element mass fraction, $Z$, have been determined from $[\mathrm{Fe} / \mathrm{H}]$ and $[\alpha / \mathrm{Fe}]$, and stellar masses from the $\log T_{\text {eff }}-\log g$ diagram by interpolating between the $\mathrm{Y}^{2}$ (Yi et al. 2003) evolutionary tracks. For the large majority of stars with $0.7<M / M_{\odot}<0.9$ and $0.001 \lesssim Z<0.006$ (corresponding to $-1.5 \lessgtr[\mathrm{Fe} / \mathrm{H}] \lessgtr-0.7)$, the lithium abundance depends quasi-linearly on mass and $Z$ as given in Eq. (2). It remains to be seen if stellar models dealing with lithium destruction and depletion can reproduce this derived relation. Furthermore, it is an open question why a few stars have a much lower lithium abundance than predicted by Eq. (2).

Extrapolating the derived relation between $A(\mathrm{Li}), M$, and $Z$ to $Z=0$ yields a lithium abundance close to the value predicted from standard BBN calculations and the WMAP baryon density. It is, therefore, tempting to suggest that halo stars with metallicities $0.001 \lesssim Z<0.006$ and masses $0.7<M / M_{\odot}<0.9$ formed with a lithium abundance close to the primordial value and that lithium in their atmospheres has later been depleted with an almost linear dependence on mass and $Z$. This suggests that the solution of the "lithium problem" is to be obtained by getting a better understanding of how lithium in the atmospheres of metal-poor F and $\mathrm{G}$ main-sequence stars is depleted by diffusion and mixing processes. It seems unlikely that the solution is to be found in terms of non-standard BBN or astration of lithium in an early generation of massive stars.

For stars more metal-poor than $Z \simeq 0.001$, the lithium abundance is lower than predicted by Eq. (2), and it is puzzling that stars with $-2.5<[\mathrm{Fe} / \mathrm{H}]<-1.5$ on the Spite plateau have the same $\mathrm{Li}$ abundance for masses in the range $0.75<M / M_{\odot}<0.82$ (Meléndez et al. 2010, Fig. 5). For stars with $[\mathrm{Fe} / \mathrm{H}]<-2.5$, the lithium abundance seems to vary from star to star depending perhaps on stellar mass (Meléndez et al. 2010). High-precision homogeneous values of $A(\mathrm{Li}), M$, and $Z$ are needed for a larger sample of stars spanning the whole metallicity range of the Galactic halo, in order to learn more about depletion of lithium in metal-poor stars.

As a main result of this work, we find no significant difference in the lithium abundance of stars probably formed in situ in the inner parts of the Galaxy and stars accreted from dwarf galaxies. This underlines the universality of lithium abundances. Metal-rich halo stars were apparently formed with the same initial $\mathrm{Li}$ abundance, and in the course of their evolution the atmospheric Li content has been depleted at a rate depending primarily on stellar mass and metallicity.

Acknowledgements. We thank the referee, Iván Ramírez, for very useful suggestions leading to improvements of this paper and Jorge Meléndez for sending an updated version of Table 1 in Meléndez et al. (2010). This publication made use of the SIMBAD database operated at CDS, Strasbourg, France, and NASA's Astrophysics Data System.

\section{References}

Aoki, W., Barklem, P. S., Beers, T. C., et al. 2009, ApJ, 698, 1803 Asplund, M., Lambert, D. L., Nissen, P. E., Primas, F., \& Smith, V. V. 2006, ApJ, 644, 229

Asplund, M., Grevesse, N., Sauval, A. J., \& Scott, P. 2009, ARA\&A, 47, 481

Barklem, P. S., Belyaev, A. K., \& Asplund, M. 2003, A\&A, 409, L1

Baumann, P., Ramírez, I., Meléndez, J., Asplund, M., \& Lind, K. 2010, A\&A, 519, A87

Boesgaard, A. M., Stephens, A., \& Deliyannis, C. P. 2005, ApJ, 633, 398

Bonifacio, P., Molaro, P., Sivarani, T., et al. 2007, A\&A, 462, 851

Carney, B. W., Latham, D. W., \& Laird, J. B. 2005, AJ, 129, 466

Casagrande, L., Ramírez, I., Meléndez, J., Bessell, M., \& Asplund, M. 2010, A\&A, 512, A54

Chen, Y. Q., Nissen, P. E., Benoni, T., \& Zhao, G. 2001, A\&A, 371, 943

Coc, A., Goriely, S., Xu, Y., Saimpert, M., \& Vangioni, E. 2012, ApJ, 744, 158

Cyburt, R. H., Fields, B. D., \& Olive, K. A. 2008, JCAP, 11, 12

Deliyannis, C. P., Demarque, P., \& Kawaler, S. D. 1990, ApJS, 73, 21

Ducati, J. R., Penteado, E. M., \& Turcati, R. 2011, A\&A, 525, A26

ESA 1997, The Hipparcos and Tycho Catalogues, ESA SP, 1200

Fields, B. D. 2011, Ann. Rev. Nucl. Part. Sci., 61, 47

González Hernández, J. I., \& Bonifacio, P. 2009, A\&A, 497, 497

Grevesse, N., \& Noels, A. 1993, in Origin and Evolution of the Elements, ed. N. Prantzos, E. Vangioni-Flam, \& M. Cassé (Cambridge Univ. Press), 15 Gustafsson, B., Edvardsson, B., Eriksson, K., et al. 2008, A\&A, 486, 951

Kim, Y. -C., Demarque, P., Yi, S. K., \& Alexander, D. R. 2002, ApJS, 143, 499 Komatsu, E., Smith, K. M., Dunkley, J., et al. 2011, ApJS, 192, 18

Lambert, D. L., \& Reddy, B. E. 2004, MNRAS, 349, 757

Latham, D. W., Stefanik, R. P., Torres, G., et al. 2002, AJ, 124, 1144

Lind, K., Asplund, M., \& Barklem, P. S. 2009, A\&A, 503, 541

Liu, S., Nissen, P. E., Schuster, W. J., et al. 2012, A\&A, 541, A48

Meléndez, J., Casagrande, L., Ramírez, I., Asplund, M., \& Schuster, W. J. 2010, A\&A, 515, L3

Molaro, P., Primas, F., \& Bonifacio, P. 1995, A\&A, 295, L47

Mowlavi, N., Eggenberger, P., Meynet, G., et al. 2012, A\&A, 541, A41

Nissen, P. E., \& Schuster, W. J. 2010, A\&A, 511, L10 (Paper I)

Nissen, P. E., \& Schuster, W. J. 2011, A\&A, 530, A15 (Paper II)

Pettini, M., Zych, B. J., Murphy, M. T., Lewis, A., \& Steidel, C. C. 2008, MNRAS, 391, 1499

Piau, L. 2008, ApJ, 689, 1279

Piau, L., Beers, T. C., Balsara, D. S., et al. 2006, ApJ, 653, 300

Pinsonneault, M. H., Walker, T. P., Steigman, G., \& Narayanan, V. K. 1999, ApJ, 527,180

Prantzos, N. 2007, Space Sci. Rev., 130, 27

Prantzos, N. 2012, A\&A, 542, A67

Purcell, C. W., Bullock, J. S., \& Kazantzidis, S. 2010, MNRAS, 404, 1711 
A\&A 543, A28 (2012)

Ramírez, I, \& Meléndez, J. 2005, ApJ, 626, 465

Richard, O., Michaud, G., \& Richer, J. 2002, ApJ, 580, 1100

Richard, O., Michaud, G., \& Richer, J. 2005, ApJ, 619, 538

Ryan, S. G., \& Deliyannis, C. P. 1998, ApJ, 500, 398

Ryan, S. G., Norris, J. E., \& Beers, T. C. 1999, ApJ, 523, 654

Salaris, M., \& Weiss, A. 2001, A\&A, 376, 955

Sansonetti, C. J., Richou, B., Engleman, R., \& Radziemski, L. J. 1995, Phys. Rev. A., 52, 2682

Sbordone, L., Bonifacio, P., Caffau, E., et al. 2010, A\&A, 522, A26

Schuster, W. J., Moreno, E., Nissen, P. E., \& Pichardo, B. 2012, A\&A, 538, A21 (Paper III)
Smith, V. V., Lambert, D. L., \& Nissen, P. E. 1998, ApJ, 506, 405 Spite, M., \& Spite, F. 1982a, Nature, 297, 483

Spite, F., \& Spite, M. 1982b, A\&A, 115, 357

Spite, M., Francois, P., Nissen, P. E., \& Spite, F. 1996, A\&A, 307, 172

Takeda, Y., \& Takada-Hidai, M. 2011, PASJ, 63, S537

Talon, S., \& Charbonnel, C. 2004, A\&A, 418, 1051

Tan, K., \& Zhao, G. 2011, ApJ, 738, L33

Yan, Z. -C., \& Drake, G. W. F. 1995, Phys. Rev. A, 52, 4316

Yi, S. K., Kim, Y. -C., \& Demarque, P. 2003, ApJS, 144, 259

Zolotov, A., Willman, B., Brooks, A. M., et al. 2009, ApJ, 702, 1058

Zolotov, A., Willman, B., Brooks, A. M., et al. 2010, ApJ, 721, 738

Pages 9 to 10 are available in the electronic edition of the journal at http://www . aanda.org 
P. E. Nissen and W. J. Schuster: Lithium abundances in two distinct halo populations

Table 1. Stellar parameters and lithium abundances derived from the equivalent width (EW) of the Li I $6707.8 \AA$ line.

\begin{tabular}{|c|c|c|c|c|c|c|c|c|c|c|c|}
\hline ID & Pop. ${ }^{a}$ & $\begin{array}{l}T_{\text {eff }} \\
{[\mathrm{K}]}\end{array}$ & $\log g$ & {$[\mathrm{Fe} / \mathrm{H}]$} & {$[\alpha / \mathrm{Fe}]$} & $Z$ & $M / M_{\odot}$ & $\begin{array}{c}E W \\
{[\mathrm{~m} \AA]}\end{array}$ & $\begin{array}{r}A(\mathrm{Li}) \\
\mathrm{LTE}\end{array}$ & $\begin{array}{r}A(\mathrm{Li}) \\
\text { NLTE }\end{array}$ & Note $^{b}$ \\
\hline BD-213420 & $\mathrm{C}$ & 5908 & 4.26 & -1.13 & 0.31 & 0.0024 & 0.77 & 21.2 & 1.95 & 1.92 & \\
\hline CD-33 3337 & $\mathrm{C}$ & 6079 & 3.86 & -1.36 & 0.30 & 0.0014 & 0.85 & 39.2 & 2.37 & 2.32 & \\
\hline CD-43 6810 & A & 6045 & 4.26 & -0.43 & 0.23 & 0.0105 & 0.99 & $<1.0$ & $<0.72$ & $<0.70$ & \\
\hline CD-45 3283 & B & 5697 & 4.55 & -0.91 & 0.12 & 0.0029 & 0.79 & $<1.0$ & $<0.43$ & $<0.43$ & \\
\hline CD-51 4628 & B & 6253 & 4.31 & -1.30 & 0.22 & 0.0014 & 0.85 & 29.7 & 2.36 & 2.29 & \\
\hline CD-57 1633 & B & 5973 & 4.28 & -0.90 & 0.07 & 0.0028 & 0.81 & 30.5 & 2.19 & 2.15 & \\
\hline CD-61 282 & B & 5859 & 4.31 & -1.23 & 0.22 & 0.0016 & 0.73 & 36.7 & 2.18 & 2.14 & \\
\hline G05-19 & B & 5954 & 4.26 & -1.18 & 0.19 & 0.0017 & 0.76 & 32.7 & 2.20 & 2.15 & \\
\hline G05-36 & $\mathrm{A}$ & 6113 & 4.23 & -1.23 & 0.35 & 0.0020 & 0.83 & 41.6 & 2.43 & 2.37 & \\
\hline G05-40 & A & 5895 & 4.17 & -0.81 & 0.31 & 0.0049 & 0.83 & 13.5 & 1.74 & 1.72 & \\
\hline G13-38 & A & 5363 & 4.54 & -0.88 & 0.32 & 0.0044 & 0.73 & $<3.0$ & $<0.58$ & $<0.61$ & \\
\hline G15-23 & A & 5397 & 4.57 & -1.10 & 0.34 & 0.0028 & 0.71 & $<3.0$ & $<0.61$ & $<0.63$ & \\
\hline G16-20 & B & 5725 & 3.64 & -1.42 & 0.26 & 0.0011 & 0.85 & 38.8 & 2.10 & 2.07 & \\
\hline G18-28 & A & 5472 & 4.41 & -0.83 & 0.31 & 0.0048 & 0.72 & $<2.0$ & $<0.51$ & $<0.53$ & SB1 \\
\hline G18-39 & A & 6140 & 4.21 & -1.39 & 0.34 & 0.0014 & 0.80 & 30.6 & 2.29 & 2.23 & \\
\hline G20-15 & B & 6127 & 4.32 & -1.49 & 0.24 & 0.0010 & 0.78 & 34.1 & 2.33 & 2.27 & \\
\hline G21-22 & B & 6001 & 4.24 & -1.09 & 0.09 & 0.0018 & 0.78 & 37.9 & 2.31 & 2.25 & \\
\hline G24-13 & A & 5773 & 4.31 & -0.72 & 0.29 & 0.0059 & 0.82 & $<3.0$ & $<0.97$ & $<0.97$ & \\
\hline G24-25 & A & 5928 & 3.86 & -1.40 & 0.35 & 0.0014 & 0.81 & $<2.0$ & $<0.90$ & $<0.87$ & SB1 \\
\hline G31-55 & A & 5738 & 4.30 & -1.10 & 0.29 & 0.0025 & 0.72 & 10.9 & 1.51 & 1.49 & \\
\hline G46-31 & B & 6001 & 4.23 & -0.83 & 0.15 & 0.0037 & 0.83 & 26.1 & 2.14 & 2.10 & SB1 \\
\hline G49-19 & A & 5872 & 4.25 & -0.55 & 0.27 & 0.0085 & 0.89 & 4.5 & 1.23 & 1.23 & SB1 \\
\hline G53-41 & B & 5959 & 4.27 & -1.20 & 0.23 & 0.0018 & 0.76 & 27.2 & 2.11 & 2.06 & \\
\hline G56-30 & B & 5930 & 4.26 & -0.89 & 0.11 & 0.0030 & 0.80 & 29.5 & 2.14 & 2.10 & \\
\hline G56-36 & B & 6033 & 4.28 & -0.94 & 0.20 & 0.0032 & 0.84 & 24.1 & 2.12 & 2.07 & \\
\hline G57-07 & A & 5776 & 4.25 & -0.47 & 0.31 & 0.0107 & 0.90 & $<2.0$ & $<0.80$ & $<0.81$ & \\
\hline G63-26 & A & 6143 & 4.18 & -1.56 & 0.37 & 0.0010 & 0.78 & 46.8 & 2.50 & 2.43 & \\
\hline G66-22 & B & 5336 & 4.41 & -0.86 & 0.12 & 0.0033 & 0.64 & 10.8 & 1.12 & 1.16 & \\
\hline G74-32 & A & 5872 & 4.36 & -0.72 & 0.30 & 0.0060 & 0.86 & $<3.0$ & $<1.05$ & $<1.04$ & \\
\hline G75-31 & B & 6110 & 4.02 & -1.03 & 0.20 & 0.0025 & 0.87 & 40.1 & 2.42 & 2.36 & \\
\hline G81-02 & A & 5959 & 4.19 & -0.69 & 0.19 & 0.0054 & 0.86 & 25.2 & 2.09 & 2.06 & \\
\hline G82-05 & B & 5377 & 4.45 & -0.75 & 0.09 & 0.0040 & 0.68 & 8.6 & 1.04 & 1.08 & \\
\hline G85-13 & A & 5728 & 4.38 & -0.59 & 0.28 & 0.0079 & 0.86 & $<2.0$ & $<0.75$ & $<0.76$ & \\
\hline G87-13 & B & 6185 & 4.13 & -1.09 & 0.20 & 0.0022 & 0.86 & 44.1 & 2.52 & 2.45 & \\
\hline G94-49 & A & 5473 & 4.50 & -0.80 & 0.31 & 0.0051 & 0.76 & $<3.0$ & $<0.69$ & $<0.71$ & \\
\hline G96-20 & A & 6393 & 4.41 & -0.89 & 0.28 & 0.0040 & 1.00 & $<2.0$ & $<1.26$ & $<1.21$ & \\
\hline G98-53 & B & 5948 & 4.23 & -0.87 & 0.19 & 0.0035 & 0.81 & 23.6 & 2.04 & 2.01 & \\
\hline G99-21 & A & 5587 & 4.39 & -0.67 & 0.29 & 0.0068 & 0.79 & $<3.0$ & $<0.80$ & $<0.82$ & \\
\hline G112-43 & B & 6174 & 4.03 & -1.25 & 0.24 & 0.0016 & 0.84 & 41.9 & 2.48 & 2.41 & \\
\hline G112-44 & B & 5919 & 4.25 & -1.29 & 0.22 & 0.0014 & 0.73 & 43.3 & 2.31 & 2.25 & \\
\hline G114-42 & B & 5743 & 4.38 & -1.10 & 0.19 & 0.0021 & 0.72 & 24.3 & 1.88 & 1.86 & \\
\hline G119-64 & B & 6281 & 4.18 & -1.48 & 0.28 & 0.0010 & 0.82 & 32.3 & 2.41 & 2.35 & \\
\hline G121-12 & B & 6028 & 4.23 & -0.93 & 0.10 & 0.0027 & 0.82 & 43.0 & 2.40 & 2.35 & \\
\hline G125-13 & A & 5948 & 4.28 & -1.43 & 0.27 & 0.0011 & 0.73 & 38.0 & 2.26 & 2.20 & \\
\hline G127-26 & A & 5891 & 4.14 & -0.53 & 0.24 & 0.0085 & 0.91 & 17.6 & 1.87 & 1.86 & \\
\hline G150-40 & B & 6068 & 4.09 & -0.81 & 0.16 & 0.0040 & 0.88 & 28.2 & 2.22 & 2.18 & \\
\hline G159-50 & A & 5724 & 4.37 & -0.93 & 0.31 & 0.0038 & 0.76 & 5.2 & 1.16 & 1.16 & \\
\hline G161-73 & B & 6086 & 4.00 & -1.00 & 0.16 & 0.0026 & 0.87 & 44.3 & 2.46 & 2.39 & \\
\hline G170-56 & B & 6094 & 4.12 & -0.92 & 0.17 & 0.0031 & 0.86 & 32.9 & 2.31 & 2.26 & \\
\hline G172-61 & B & 5325 & 4.47 & -1.00 & 0.19 & 0.0026 & 0.63 & $<4.0$ & $<0.67$ & $<0.70$ & SB1 \\
\hline G176-53 & B & 5623 & 4.48 & -1.34 & 0.18 & 0.0012 & 0.67 & 24.6 & 1.79 & 1.77 & \\
\hline G180-24 & A & 6104 & 4.21 & -1.39 & 0.33 & 0.0013 & 0.79 & 32.9 & 2.30 & 2.24 & \\
\hline G187-18 & A & 5707 & 4.39 & -0.67 & 0.26 & 0.0065 & 0.82 & $<2.0$ & $<0.73$ & $<0.74$ & \\
\hline G188-22 & A & 6074 & 4.18 & -1.32 & 0.35 & 0.0017 & 0.79 & 35.2 & 2.32 & 2.26 & \\
\hline G192-43 & B & 6270 & 4.29 & -1.34 & 0.26 & 0.0014 & 0.85 & 28.9 & 2.35 & 2.29 & \\
\hline G232-18 & A & 5659 & 4.48 & -0.93 & 0.32 & 0.0039 & 0.78 & $<3.0$ & $<0.87$ & $<0.86$ & \\
\hline HD 3567 & B & 6151 & 4.02 & -1.16 & 0.21 & 0.0019 & 0.85 & 38.3 & 2.42 & 2.36 & \\
\hline HD 22879 & $\mathrm{C}$ & 5859 & 4.25 & -0.85 & 0.31 & 0.0046 & 0.81 & 10.4 & 1.59 & 1.57 & \\
\hline HD 25704 & $\mathrm{C}$ & 5968 & 4.26 & -0.85 & 0.24 & 0.0041 & 0.83 & 21.5 & 2.02 & 1.98 & D \\
\hline HD 51754 & A & 5867 & 4.29 & -0.58 & 0.26 & 0.0078 & 0.88 & 3.8 & 1.15 & 1.15 & \\
\hline HD 59392 & B & 6112 & 3.91 & -1.60 & 0.32 & 0.0008 & 0.80 & 39.2 & 2.38 & 2.33 & \\
\hline HD 76932 & $\mathrm{C}$ & 5977 & 4.13 & -0.87 & 0.29 & 0.0043 & 0.85 & 24.9 & 2.09 & 2.06 & \\
\hline HD 97320 & C & 6108 & 4.19 & -1.17 & 0.28 & 0.0021 & 0.82 & 39.7 & 2.41 & 2.35 & \\
\hline
\end{tabular}


Table 1. continued.

\begin{tabular}{|c|c|c|c|c|c|c|c|c|c|c|c|}
\hline ID & Pop. ${ }^{a}$ & $\begin{array}{l}T_{\text {eff }} \\
{[\mathrm{K}]}\end{array}$ & $\log g$ & {$[\mathrm{Fe} / \mathrm{H}]$} & {$[\alpha / \mathrm{Fe}]$} & $Z$ & $M / M_{\odot}$ & $\begin{array}{r}E W \\
{[\mathrm{~m} \AA]}\end{array}$ & $\begin{array}{r}A(\mathrm{Li}) \\
\mathrm{LTE}\end{array}$ & $\begin{array}{r}A(\mathrm{Li}) \\
\text { NLTE }\end{array}$ & Note $^{b}$ \\
\hline HD 103723 & $\mathrm{~B}$ & 6038 & 4.19 & -0.80 & 0.14 & 0.0039 & 0.85 & 30.4 & 2.24 & 2.20 & \\
\hline HD 105004 & B & 5854 & 4.30 & -0.82 & 0.14 & 0.0036 & 0.79 & 19.3 & 1.88 & 1.86 & \\
\hline HD 106516 & $\mathrm{C}$ & 6296 & 4.42 & -0.68 & 0.29 & 0.0066 & 1.03 & $<1.0$ & $<0.89$ & $<0.86$ & SB1 \\
\hline HD 111980 & A & 5878 & 3.96 & -1.08 & 0.34 & 0.0028 & 0.82 & 49.0 & 2.35 & 2.30 & SB1 \\
\hline HD 113679 & A & 5772 & 3.99 & -0.65 & 0.32 & 0.0074 & 0.88 & 34.0 & 2.08 & 2.07 & \\
\hline HD $114762 \mathrm{~A}$ & $\mathrm{C}$ & 5956 & 4.21 & -0.70 & 0.24 & 0.0056 & 0.87 & 25.5 & 2.09 & 2.06 & SB1 \\
\hline HD 120559 & $\mathrm{C}$ & 5512 & 4.50 & -0.89 & 0.30 & 0.0041 & 0.74 & $<2.0$ & $<0.55$ & $<0.57$ & \\
\hline HD 121004 & A & 5769 & 4.37 & -0.70 & 0.32 & 0.0065 & 0.84 & $<3.0$ & $<0.96$ & $<0.96$ & \\
\hline HD 126681 & $\mathrm{C}$ & 5607 & 4.45 & -1.17 & 0.35 & 0.0023 & 0.71 & 14.5 & 1.52 & 1.51 & \\
\hline HD 132475 & A & 5746 & 3.76 & -1.49 & 0.38 & 0.0012 & 0.78 & 53.4 & 2.28 & 2.23 & \\
\hline HD 148816 & A & 5923 & 4.13 & -0.73 & 0.27 & 0.0056 & 0.86 & 18.9 & 1.92 & 1.90 & \\
\hline HD 159482 & A & 5837 & 4.31 & -0.73 & 0.30 & 0.0060 & 0.83 & $<2.0$ & $<0.85$ & $<0.84$ & D \\
\hline HD 160693 & A & 5814 & 4.27 & -0.49 & 0.19 & 0.0087 & 0.87 & 6.4 & 1.34 & 1.35 & \\
\hline HD 163810 & B & 5601 & 4.56 & -1.20 & 0.21 & 0.0017 & 0.73 & 22.3 & 1.72 & 1.71 & $\mathrm{D}$ \\
\hline HD 175179 & $\mathrm{C}$ & 5813 & 4.33 & -0.65 & 0.29 & 0.0069 & 0.85 & $<2.0$ & $<0.83$ & $<0.83$ & \\
\hline HD 177095 & A & 5449 & 4.39 & -0.74 & 0.31 & 0.0060 & 0.73 & $<3.0$ & $<0.67$ & $<0.70$ & \\
\hline HD 179626 & A & 5950 & 4.13 & -1.04 & 0.31 & 0.0030 & 0.80 & 24.1 & 2.05 & 2.01 & \\
\hline HD 189558 & $\mathrm{C}$ & 5717 & 3.80 & -1.12 & 0.33 & 0.0025 & 0.83 & 58.9 & 2.31 & 2.27 & \\
\hline HD 193901 & B & 5756 & 4.36 & -1.09 & 0.16 & 0.0020 & 0.72 & 28.1 & 1.97 & 1.94 & \\
\hline HD 194598 & B & 6042 & 4.33 & -1.09 & 0.18 & 0.0021 & 0.82 & 32.9 & 2.27 & 2.21 & \\
\hline HD 199289 & $\mathrm{C}$ & 5910 & 4.28 & -1.04 & 0.30 & 0.0029 & 0.79 & 17.9 & 1.88 & 1.84 & \\
\hline HD 205650 & $\mathrm{C}$ & 5798 & 4.32 & -1.17 & 0.30 & 0.0021 & 0.73 & 14.8 & 1.70 & 1.67 & \\
\hline HD 219617 & B & 5962 & 4.28 & -1.45 & 0.28 & 0.0011 & 0.73 & 37.3 & 2.26 & 2.20 & D \\
\hline HD 222766 & A & 5434 & 4.27 & -0.67 & 0.30 & 0.0069 & 0.72 & $<2.0$ & $<0.47$ & $<0.51$ & \\
\hline HD 230409 & A & 5418 & 4.54 & -0.85 & 0.27 & 0.0043 & 0.74 & $<2.0$ & $<0.46$ & $<0.49$ & \\
\hline HD 233511 & A & 6106 & 4.23 & -1.55 & 0.34 & 0.0010 & 0.76 & 33.0 & 2.30 & 2.24 & \\
\hline HD 237822 & A & 5703 & 4.33 & -0.45 & 0.29 & 0.0110 & 0.90 & $<2.0$ & $<0.73$ & $<0.75$ & \\
\hline HD 241253 & $\mathrm{C}$ & 5931 & 4.31 & -1.10 & 0.29 & 0.0025 & 0.79 & 19.3 & 1.93 & 1.89 & \\
\hline HD 250792A & B & 5589 & 4.47 & -1.01 & 0.24 & 0.0028 & 0.72 & 15.6 & 1.54 & 1.54 & D \\
\hline HD 284248 & B & 6235 & 4.25 & -1.57 & 0.27 & 0.0008 & 0.80 & 31.5 & 2.36 & 2.30 & \\
\hline
\end{tabular}

Notes. ${ }^{(a)}$ Population classification: A, high-alpha halo; B, low-alpha halo; C, thick disk. ${ }^{(b)}$ Note on binarity: SB1, single-lined spectroscopic binary; D, double star. 